\title{
MODELADO Y SIMULACION DE UN AEROGENERADOR DE 5 MW ESCALADO A PARTIR DE UNO DE 7 KW
}

\author{
Laura Olaya Rúa \\ Facultad de Físicas, Universidad Complutense de Madrid, lolaya@ucm.es \\ Jesús Enrique Sierra-García \\ Universidad de Burgos, jesierra@ubu.es \\ Matilde Santos Peñas \\ Instituto de Tecnología del Conocimiento, Universidad Complutense de Madrid, msantos@ucm.es
}

\begin{abstract}
Resumen
En este trabajo se diseña e implementa una metodología para escalar el modelo de una turbina eólica de potencia nominal determinada al de una turbina de diferente potencia. Para ello, se presentan e implementan en MATLAB/Simulink los modelos que describen el funcionamiento de un aerogenerador, se identifican los parámetros que definen su potencia nominal, y se enumeran y aplican las modificaciones que deben realizarse para escalar un modelo a otro. Los resultados de simulación confirman la viabilidad de esta metodología, que puede resultar muy útil para obtener de forma sistemática y más sencilla el modelo de las más complejas turbinas de gran tamaño.
\end{abstract}

Palabras clave: energía eólica, turbina eólica, modelado y simulación, escalamiento, potencia.

\section{INTRODUCCIÓN}

Las turbinas eólicas pueden ser instaladas en diferentes entornos, dependiendo de su capacidad. Los aerogeneradores pequeños, con una potencia nominal de menos de $50 \mathrm{~kW}$, normalmente se diseñan para el autoabastecimiento, uso residencial y algunas industrias no muy grandes. Los aerogeneradores grandes, con una potencia de megavatios, son empleados en la producción de energía para la red eléctrica.

Este trabajo busca identificar los factores que definen la potencia de una turbina eólica y diseñar una metodología para escalar el modelo de una turbina dada a una potencia deseada.

Contar con modelos de aerogeneradores de diferentes capacidades puede ser de utilidad en múltiples aplicaciones. En el trabajo de Coutinho et al. [3], los autores demuestran la importancia del modelado numérico de aerogeneradores para predecir el comportamiento de los mismos ante las cargas a las que están sometidos, incluso antes de su implantación en parques eólicos. Emplean el programa GH Bladed 4.5 para simular dos turbinas de capacidades de $2 \mathrm{MW}$ y $5 \mathrm{MW}$, con el fin de comparar su respuesta. Los autores Burmester et al. [1] comparan los coeficientes de sustentación y de arrastre de un prototipo de aerogenerador, obtenidos mediante simulación, con los datos obtenidos durante el funcionamiento de un aerogenerador real. Khan [8] realiza el modelado, simulación y análisis de una pequeña turbina aislada, mediante conjuntos de relaciones empíricas y físicas, además de su simulación digital.

En la industria se cuenta con herramientas de simulación de aerogeneradores de diferentes capacidades. Sin embargo, su adquisición puede ser costosa, además de que el tiempo de formación requerido para su uso puede ser muy largo. En este estudio se hace uso de las herramientas MATLAB/Simulink para obtener modelos definidos por un conjunto de ecuaciones de fácil comprensión y modificación.

El escalamiento de modelos de aerogeneradores, entre otros beneficios, puede ser de utilidad para para predecir el comportamiento de turbinas de diferentes capacidades, comparar el comportamiento de prototipos frente a su contraparte real, y aprovechar medidas empíricas realizadas en una turbina de capacidad determinada para validar un modelo. Por ello, en este trabajo se propone un procedimiento para escalar un modelo de una turbina de $5 \mathrm{MW}$ a partir de uno de $7 \mathrm{~kW}$.

La metodología es versátil, de manera que permite obtener modelos de turbinas de mayor capacidad o más pequeñas, como la de un prototipo.

Esta metodología propuesta se ha validado realizando el escalamiento del modelo de una turbina de $7 \mathrm{~kW}$ desarrollado en [10] a una de mayor capacidad. En concreto, considerando la situación actual de la energía eólica, donde se tiende a la instalación de grandes turbinas offshore para hacerlas más rentables [16], se ha decidido escalar este modelo a una turbina 
de 5 MW. Para ello se usa como base la turbina de 5 MW, desarrollada por el Laboratorio Nacional de Energía Renovable (NREL) [7].

Los resultados se han validado con los valores esperados obtenidos con la simulación de la turbina objetivo mediante un software específico de alta precisión, FAST. Estos resultados confirman la viabilidad de esta metodología que puede resultar muy útil para obtener de forma sistemática y más sencilla el modelo de turbinas de gran tamaño.

A continuación, se detalla la organización del documento. En la sección 2 se describen los subsistemas de un aerogenerador, identificando las ecuaciones más relevantes. La sección 3 recoge los parámetros de la turbina de 5MW escalada desde la turbina de $7 \mathrm{~kW}$. En la sección 4 se muestran los resultados de simulación del modelo escalado. El documento finaliza con las conclusiones y trabajos futuros.

\section{DESCRIPCIÓN DE LOS SUBSISTEMAS DE LA TURBINA}

Con el fin de facilitar las modificaciones que se deben realizar en el modelo, el aerogenerador se divide en los siguientes subsistemas: el viento, la potencia, la relación de engranajes, y el generador.

\subsection{El viento capturado}

Uno de los principales parámetros que diferencian a una turbina pequeña de una grande es el radio de su rotor, cuyo impacto se ve reflejado en la siguiente expresión [2].

$$
\rho A_{\infty} v_{\infty}=\rho A_{r} v_{r}=\rho A_{w} v_{w}
$$

Donde A es el área de barrido $\left[\mathrm{m}^{2}\right], v$ es la velocidad del viento $[\mathrm{m} / \mathrm{s}]$, el subíndice $\infty$ se refiere a los parámetros del viento libre antes de interactuar con el rotor, el subíndice $r$ re refiere a los parámetros a nivel del rotor, y $w$ a los parámetros de la estela de viento que sale del rotor. La ecuación (1) describe la relación entre el caudal másico del flujo de aire antes, durante y después de su paso por el rotor. Al igual que sucede en una tubería sin pérdidas, el caudal másico en la entrada debe ser el mismo que en la salida. Por otro lado, la velocidad del viento disminuye a lo largo de su trayecto por el tubo de viento, debido a su interacción con el rotor [2]. Para mantener el caudal, el área aumenta a lo largo del tubo de viento. Esta área sólo se conoce a nivel del rotor y depende directamente de su radio.

En las turbinas grandes se busca que la captura del viento sea máxima a velocidades de viento menores a la velocidad nominal, con el fin de maximizar la generación de potencia. Una vez alcanzada la potencia nominal, ésta debe mantenerse constante para garantizar una salida estable de potencia eléctrica. Esto se consigue mediante el control de ángulo de cabeceo o control de pitch [14].

El ángulo de pitch $\theta\left[{ }^{\circ}\right]$ representa la rotación de las palas del rotor, que permite variar el ángulo entre las componentes de la velocidad de viento $\phi\left[{ }^{\circ}\right]$ y el ángulo de ataque del viento $\alpha\left[^{\circ}\right]$ [4] (Figura 1)

$$
\begin{aligned}
\tan \phi & =\frac{(1-a) v}{\left(1+a^{\prime}\right) \omega r} \\
\alpha & =\phi-\theta
\end{aligned}
$$

Donde $\omega[\mathrm{rad} / \mathrm{s}]$ es la velocidad angular del rotor, representada en la Figura 1 como $\Omega$. Tanto $a$ como $a^{\prime}$ son factores de aprovechamiento de la velocidad del viento a nivel del rotor.

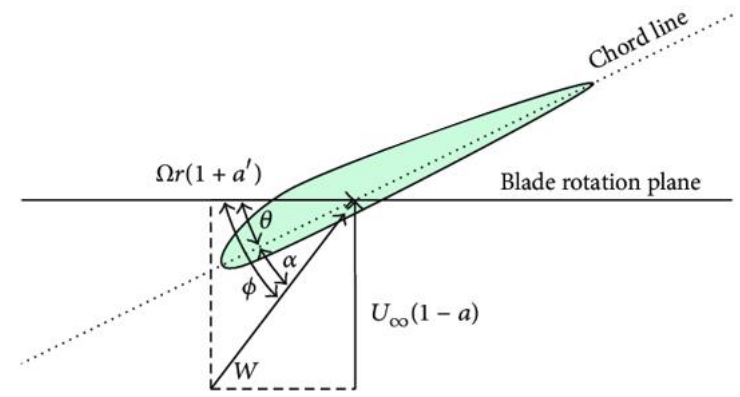

Figura 1: Triángulo de velocidades para una sección de pala [6].

Generalmente los aerogeneradores pequeños no implementan control de pitch dado que, al ser de uso residencial, no se requiere que la salida de potencia eléctrica sea tan estable. Cuando se supera el valor de viento nominal se realiza un plegado total de las palas por seguridad.

El ángulo de pitch suele estar tabulado respecto a la potencia de salida que se genera frente a una velocidad de viento determinada. Este puede ser implementado de distintas formas, como una tabla de búsqueda o un lazo de control [15].

\subsection{La potencia}

La aerodinámica del rotor se representa mediante las relaciones estáticas de la Teoría del Elemento de Pala [4], donde $P_{v}[\mathrm{~W}]$ denota la potencia disponible en el viento en ausencia del rotor, y $P[\mathrm{~W}]$ la potencia del aerogenerador una vez impulsado por el viento.

$$
\begin{gathered}
P_{v}=\frac{1}{2} \rho A v^{3} \\
P=P_{v} C_{p}
\end{gathered}
$$


La ecuación 5 indica que el coeficiente de potencia del aerogenerador $(C p)$ actúa como una eficiencia de extracción de potencia del viento. Igualmente se evidencia en la ecuación 4 que el área de barrido del rotor $A\left[\mathrm{~m}^{2}\right]$ tiene un impacto directo sobre la capacidad de aprovechamiento de la potencia aerodinámica [9].

El valor máximo de $C p$ está definido por el límite de Betz, el cual establece que la potencia extraída del viento no puede ser superior al $59,3 \%$ de la potencia disponible en el volumen de aire a nivel del rotor [5]. El $C p$ se define como la relación entre la potencia de viento capturada y la potencia disponible [12].

$$
C p=\frac{P}{\frac{1}{2} \rho A v^{3}}
$$

En la práctica se busca operar con coeficientes de potencia altos. Dado que los aerogeneradores pequeños normalmente no cuentan con un sistema de control, esto sólo se consigue en el rango de velocidades de viento comprendido entre la velocidad de puesta en funcionamiento y la velocidad nominal. Sin embargo, las turbinas de gran escala se pueden mantener operativas, aunque se haya superado la velocidad de viento nominal, consiguiendo operar a altos valores de $C p$ durante más tiempo.

El $C p$ tiene una gran dependencia de la velocidad del viento. Sin embargo, dada la complejidad de la toma instantánea de medidas de velocidad de viento a nivel del rotor, éste se suele representar en función de la velocidad del extremo de la pala (TSR, por sus siglas en inglés),

$$
T S R=\lambda=\frac{\omega R}{v}
$$

Siendo $\omega[\mathrm{rad} / \mathrm{s}]$ la velocidad angular del rotor, $v[\mathrm{~m} / \mathrm{s}]$ la velocidad del viento y $R[\mathrm{~m}]$ el radio de la pala.

En el caso de la turbina NREL de $5 \mathrm{MW}$, el funcionamiento óptimo se logra a $C p=0,482$, cuando $\lambda=7,55$ [7]. La correlación $C p-\lambda$ es característica de cada tipo de aerogenerador y permite conocer el punto de operación óptimo de la turbina en todo momento. Ésta se obtiene en la industria mediante medidas empíricas o por simulaciones aerodinámicas.

Con el fin de obtener la característica $C p-\lambda$ de la turbina de 5 MW estudiada, se realiza una aproximación a la función analítica general del $C p$

$$
C p(\lambda, \theta)=c_{1}\left(c_{2} \frac{1}{\beta}-c_{3} \theta-c_{4} \theta^{x}-c_{5}\right) e^{-c_{6} \frac{1}{\beta}}
$$

$$
\frac{1}{\beta}=\frac{1}{\lambda+0,08 \theta}-\frac{0,035}{1+\theta^{3}}
$$

A partir de la base de datos existente del National Wind Technology center de la turbina de 5 MW [11], se extraen los valores de $\mathrm{Cp}$ frente a TSR para diferentes velocidades de viento, y se grafican las curvas características para ángulos de pitch de $-1^{\circ}, 0^{\circ}$ y $1^{\circ}$. Por otro lado, se extraen los parámetros de las funciones analíticas de $\mathrm{Cp}$ definidas en [10] y [12], correspondientes a aerogeneradores de $7 \mathrm{~kW}$ y $2 \mathrm{MW}$, respectivamente, para un ángulo de pitch de $0^{\circ}$, y se selecciona la función analítica más cercana a la curva esperada definida por el NREL. Finalmente se realiza un ajuste manual de los parámetros, con el fin de hacer coincidir la curva empírica con la función analítica, obteniéndose los siguientes valores: $\mathrm{c} 1=0,47$ $\mathrm{c} 2=126 \quad \mathrm{c} 3=0,3 \quad \mathrm{c} 4=0,002 \quad \mathrm{c} 5=5,5 \quad \mathrm{c} 6=19 \mathrm{y}$ el exponente es $\mathrm{x}=2$.

Como se observa en las ecuaciones (8) y (9), la característica $C p-\lambda$ depende del ángulo de pitch, demostrando que la inclinación de las palas tiene un efecto sobre la capacidad de extracción de potencia del viento del rotor. Esto se debe a que se produce una variación de la componente normal del viento sobre la superficie de las palas.

En la figura 2 se presenta la curva característica obtenida de forma analítica para un ángulo de pitch de $0^{\circ}$ (línea azul), y se compara con las curvas características para ángulos de pitch de $-1^{\circ}, 0^{\circ} \mathrm{y} 1^{\circ}$, obtenidas por NREL mediante simulaciones en FAST.

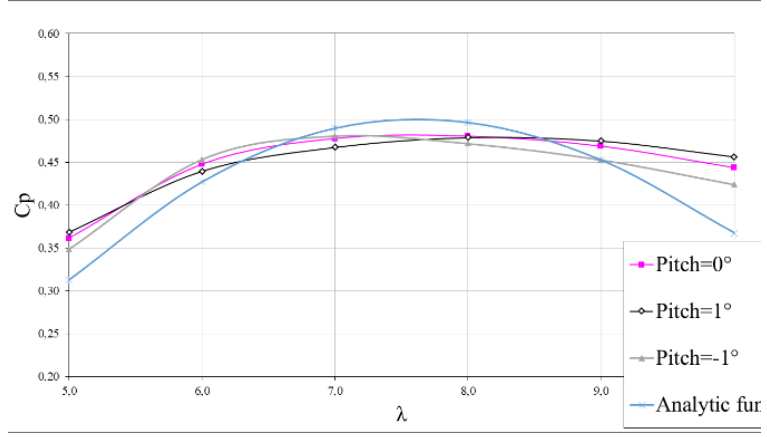

Figura 2: Representación de la característica $\mathrm{Cp}-\lambda$.

En la figura 2 se puede observar cómo la variación del ángulo de pitch produce una variación del valor del TSR al cual se obtiene el Cp máximo. En concreto, para esta turbina de $5 \mathrm{MW}$, con un pitch de $0^{\circ}$ el $\mathrm{Cp}$ máximo se obtiene en 7 y con pitch de $1^{\circ}$ es 8 .

\subsection{La relación de engranajes}

La relación de engranajes $\left(n_{g}\right)$ tiene como función multiplicar la velocidad de rotación del eje del generador con respecto a la velocidad del rotor. Los aerogeneradores pequeños suelen ser de transmisión 
directa $\left(n_{g}=1\right)$, dado que su rotor gira a velocidades suficientes para generar la potencia esperada. En aerogeneradores de gran tamaño esta relación debe ser mayor a 1 dada la alta inercia que encuentra la rotación. Al aumentar la velocidad de rotación, la relación de engranajes reduce el torque mecánico transferido al generador,

$$
\tau_{g}=\frac{1}{n_{g}} \frac{P}{\omega}
$$

La relación de engranajes se modela a partir de la segunda ley de Newton, donde el torque mecánico transmitido al generador $\left(\tau_{g}-\tau_{e m}\right)[\mathrm{Nm}]$ es función de la aceleración angular del eje de rotación y de la inercia de la masa en movimiento [12].

$$
\frac{d \omega}{d t}=\frac{\tau_{g}-\tau_{e m}}{J_{e q}}-\frac{B_{m}}{J_{e q}} \omega
$$

Siendo $\tau_{e m}[\mathrm{Nm}]$ el torque electromagnético entre los imanes permanentes del generador, que representa una pérdida del torque útil, y $B_{m}[\mathrm{Nm} /(\mathrm{rad} / \mathrm{s})]$ el coeficiente de amortiguamiento del eje del generador. $J_{e q}\left[\mathrm{~kg} \mathrm{~m}^{2}\right]$ representa la inercia equivalente del sistema rotor-generador, masa que se encuentra en movimiento,

$$
J_{e q}=J_{g}+\frac{J_{r}}{n_{g}^{2}}
$$

Donde $J_{g}\left[\mathrm{~kg} \mathrm{~m}^{2}\right]$ describe la inercia del eje giratorio del generador y $J_{r}\left[\mathrm{~kg} \mathrm{~m}^{2}\right]$ la inercia de rotación de las tres palas alrededor del buje.

$$
J_{r}=3 J_{\text {blade }}
$$

Siendo $\boldsymbol{J}_{\text {blade }}\left[\mathrm{kg} \mathrm{m}^{2}\right]$ la inercia de cada una de las palas del rotor.

\section{$2.4 \quad$ El generador}

Las turbinas de 5MW tienen habitualmente un generador síncrono de imanes permanentes (PMSG) [13], que se puede modelar mediante el circuito equivalente presentado en la Figura 3. En este circuito, el voltaje de salida del generador es equivalente a la fuerza electromotriz menos las pérdidas generadas en su armadura. Los parámetros del generador dependerán de la capacidad de la turbina.

La velocidad de rotación en el generador es la velocidad de rotación del rotor multiplicada por la relación de engranajes:

$$
\omega_{e}=n_{g} \omega
$$

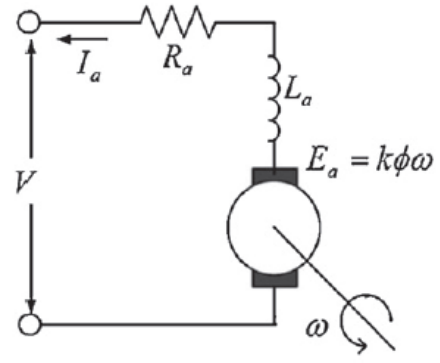

Figura 3: Circuito equivalente del PMSG.

La variación de corriente en el generador se obtiene al aplicar la ley de Kirchhoff al circuito equivalente del PMSG (Figura 3). En esta ecuación se ha supuesto que la carga $R_{L}$ es puramente resistiva.

$$
\frac{d i}{d t}=\frac{K \omega_{e}-\left(R_{a}+R_{L}\right) I}{L_{a}}
$$

Los diferentes parámetros se resuelven considerando que el aerogenerador se encuentra en estado estacionario a su potencia nominal. Para esto, se hace uso de valores esperados obtenidos con la simulación de la turbina objetivo [7], para una velocidad nominal de viento de $11.4 \mathrm{~m} / \mathrm{s}$.

Se define la constante electromotriz del generador $K$, que agrupa la constante $k$ del generador y su constante de acoplamiento magnético $\phi$, como:

$$
K=k \phi
$$

Esta constante $K$ se calcula aplicando la teoría del valor final a la ecuación (14), suponiendo que en estado estacionario la variación de corriente es nula.

De igual manera, para encontrar una expresión para el coeficiente de amortiguamiento del eje del generador $B_{m}$, se aplica la teoría del valor final a la ecuación (11), suponiendo que en estado estacionario la variación de la velocidad de rotación del rotor es nula.

Se calcula el torque electromagnético a partir de las operaciones realizadas a las ecuaciones 11 y 15.

$$
\tau_{\text {em }}=K I
$$

Se selecciona una resistencia de carga $R_{L}[\Omega]$ que permite obtener la potencia nominal a la velocidad de viento de $11.4 \mathrm{~m} / \mathrm{s}$. Con esta se calcula el voltaje de salida,

$$
V=I R_{L}
$$

A partir del voltaje y la corriente, se calcula la potencia eléctrica de salida la velocidad nominal del viento, la cual debe ser igual a la potencia nominal, 


$$
P_{\text {elec }}=V I
$$

Por último, se seleccionan valores para la resistencia e inductancia de armadura $\boldsymbol{R}_{\boldsymbol{a}}[\Omega]$ y $\boldsymbol{L}_{\boldsymbol{a}}[\mathrm{H}]$, respectivamente, que permitan conservar las igualdades establecidas por las operaciones realizadas.

\section{PARÁMETROS ESCALADOS}

A partir de la inspección de las ecuaciones (1-18) obtenemos la relación de dependencia entre todos los parámetros que intervienen en el modelo. Los resultados de este estudio se recogen en la tabla 1. Esta tabla permite evaluar el impacto de cada parámetro en el resto de los parámetros del modelo. Por ejemplo, en ella se puede observar que la velocidad del viento tiene el mayor impacto en la potencia y torque mecánico, estando elevada a la tercera potencia. La utilidad práctica de esta tabla es saber cuál es el efecto de modificar uno de los parámetros del modelo en los restantes parámetros. Por ejemplo, si doblamos el radio la potencia se multiplica por 4 , porque la relación es cuadrática.

Tabla 1: Análisis de sensibilidad de los parámetros del modelo $^{1}$

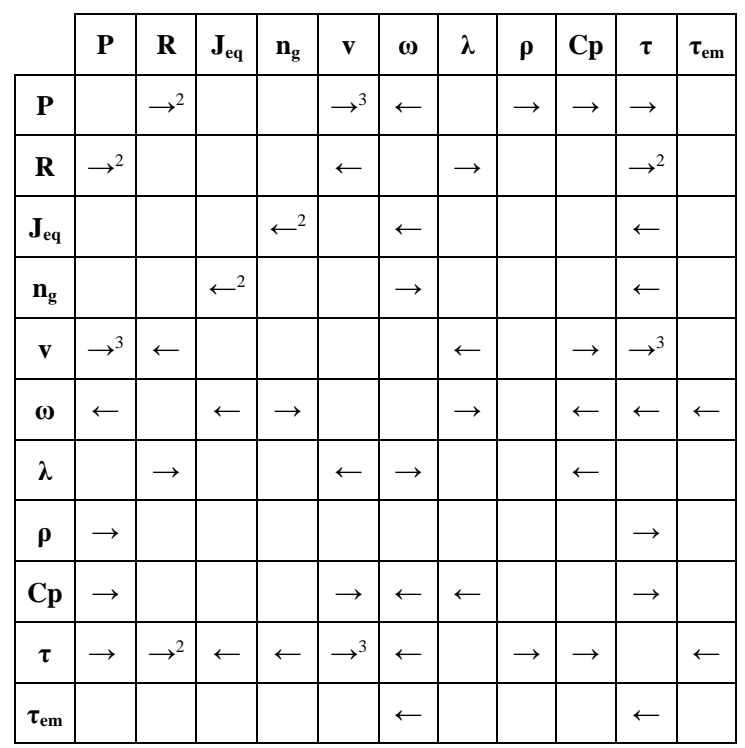

${ }^{1}$ En la tabla 1, las flechas hacia la derecha denotan una relación directa entre los parámetros relacionados por la celda y las flechas hacia la izquierda una relación inversa. La relación es lineal si no hay superíndice, cuadrática, si vale 2, o cúbica si vale 3 .

Otro resultado observable es que la velocidad de rotor y el torque mecánico son las medidas que afectan a más parámetros. Por este motivo, la representación gráfica de estas señales puede proporcionar bastante información sobre el comportamiento del aerogenerador, sin necesidad de tener conocimiento de otras variables. Además, como cabría suponer, la matriz de la tabla 1 es simétrica.

En la tabla 2 se presentan los parámetros que permiten escalar el modelo de una turbina de $7 \mathrm{~kW}$ a una de $5 \mathrm{MW}$. Los parámetros relativos al generador se obtienen mediante el procedimiento descrito en la sección 2.4. Los parámetros relativos a la turbina se obtienen de la definición de la turbina de $5 \mathrm{MW}$, desarrollada por el Laboratorio Nacional de Energía Renovable (NREL) [7]. Los valores de inercia se obtienen de [7]. Estos valores también se pueden aproximar suponiendo que las palas son rectángulos de lado igual al radio, y ancho igual a un valor de cuerda de pala promedio; y que el generador se puede aproximar mediante un cilindro cuyo radio depende de las dimensiones del generador.

En la tabla se puede observar cómo la inercia es uno de los parámetros que más varía, dado el aumento significativo en las dimensiones de ésta. La turbina pequeña cuenta con parámetros que no varían, como el ángulo de pitch y la relación de velocidades entre el rotor y el generador, lo que no es posible para una turbina grande.

Tabla 2: Parámetros que permiten escalar el modelo

\begin{tabular}{|l|c|c|c|c|}
\hline \multicolumn{2}{|c|}{ Parámetro } & $\mathbf{7 ~ k W}$ & $\mathbf{5}$ MW & Unidades \\
\hline $\begin{array}{l}\text { Potencia } \\
\text { nominal }\end{array}$ & $\mathbf{P}$ & 7 & 5300 & $\mathrm{~kW}$ \\
\hline Radio & $\mathbf{R}$ & 3.2 & 63 & $\mathrm{~m}$ \\
\hline $\begin{array}{l}\text { Inercia de } \\
\text { generador }\end{array}$ & $\mathbf{J}_{\mathbf{g}}$ & & 534116 & $\mathrm{~kg} \mathrm{~m} 2$ \\
\hline Inercia de pala & $\mathbf{J}_{\text {blade }}$ & & 11776947 & $\mathrm{~kg} \mathrm{~m} 2$ \\
\hline $\begin{array}{l}\text { Inercia } \\
\text { equivalente }\end{array}$ & $\mathbf{J}_{\text {eq }}$ & 6.525 & 898320 & $\mathrm{~kg} \mathrm{~m} 2$ \\
\hline $\begin{array}{l}\text { Relación } \\
\text { engranajes }\end{array}$ & $\mathbf{n}_{\mathbf{g}}$ & $1: 1$ & $97: 1$ & \\
\hline Ángulo de pitch & $\mathbf{\theta}_{\mathbf{b}}$ & 0 & $\mathrm{De} 0 \mathrm{a} 30$ & $\mathrm{o}$ \\
\hline $\begin{array}{l}\text { Constante } \\
\text { amortiguamiento }\end{array}$ & $\mathbf{B}_{\mathrm{m}}$ & 0.025 & 2.13 & $\mathrm{Nm} /(\mathrm{rad} / \mathrm{s})$ \\
\hline $\begin{array}{l}\text { Resistencia } \\
\text { armadura }\end{array}$ & $\mathbf{R}_{\mathrm{a}}$ & 0.275 & 0.08 & $\Omega$ \\
\hline $\begin{array}{l}\text { Resistencia } \\
\text { carga }\end{array}$ & $\mathbf{R}_{\mathrm{L}}$ & 8 & 16.1561 & $\Omega$ \\
\hline $\begin{array}{l}\text { Inductancia } \\
\text { armadura }\end{array}$ & $\mathrm{L}_{\mathrm{a}}$ & 0.0135 & 0.0135 & $\mathrm{H}$ \\
\hline $\begin{array}{l}\text { Constante } \\
\text { electromotriz }\end{array}$ & $\mathrm{K}$ & 6.15 & 52 & \\
\hline
\end{tabular}

\section{RESULTADOS DE SIMULACION DEL MODELO ESCALADO}

A continuación, se representan los resultados obtenidos en la simulación con MATLAB/Simulink de los modelos de aerogenerador de $7 \mathrm{~kW}$ y de $5 \mathrm{MW}$. 
Los modelos se someten a una entrada de viento de tipo rampa que aumenta de 0 a $27 \mathrm{~m} / \mathrm{s}$ (Figura 4). Esta señal ayuda a estudiar el comportamiento de las turbinas al sobrepasar las velocidades de puesta en funcionamiento, nominal y de corte o de frenado.

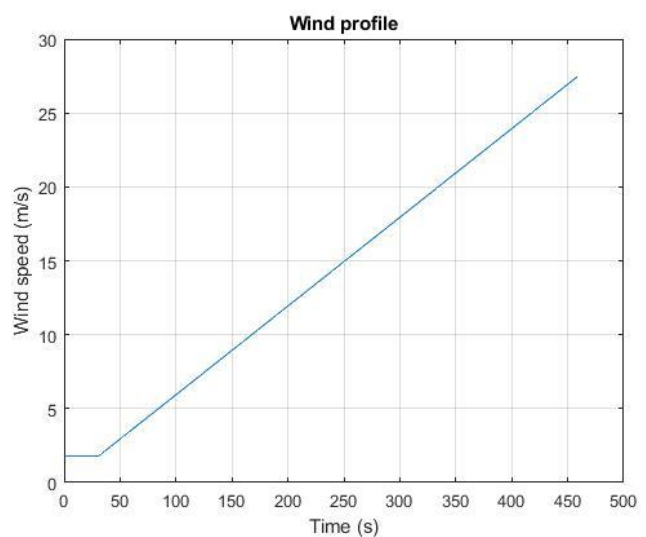

Figura 4: Perfil de viento de entrada en la simulación.

En la figura 5 se observa cómo para esta turbina de 5 MW la velocidad de viento de puesta en funcionamiento es de $3.5 \mathrm{~m} / \mathrm{s}$, la velocidad nominal a la cual la turbina alcanza su potencia nominal de 5.3 MW es $11.4 \mathrm{~m} / \mathrm{s}$, y la velocidad a la cual se frena la turbina es de $25 \mathrm{~m} / \mathrm{s}$ [7], lo cual evita que se someta a esfuerzos mecánicos elevados y hace que la turbina funcione en condiciones de seguridad.

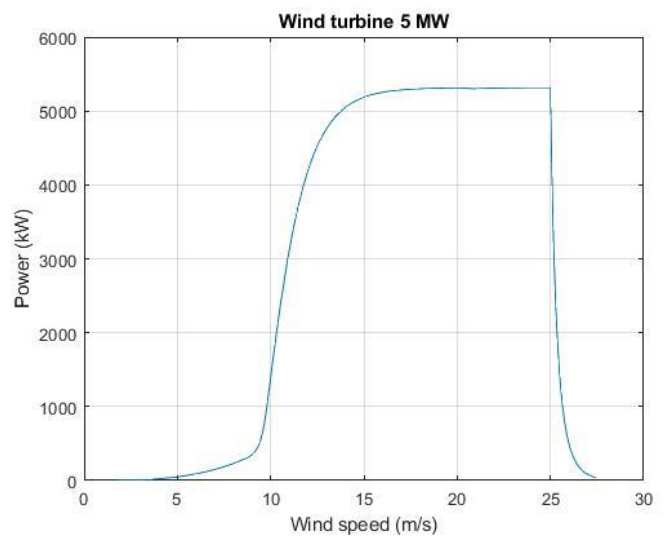

Figura 5: Potencia eléctrica de salida de la turbina de $5 \mathrm{MW}$.

Dada la capacidad de la turbina, ésta requiere un controlador de pitch para mantener la potencia nominal una vez que se alcanza la velocidad de viento de $11.4 \mathrm{~m} / \mathrm{s}$ y hasta la velocidad de corte. Por este motivo, en la figura se aprecia una meseta al alcanzar los 5.3 MW.

En la figura 6 se presenta la evolución de la potencia de una turbina de $7 \mathrm{~kW}$, considerada como pequeña en la industria. En ésta se observa como la potencia cae precipitadamente después de alcanzar la velocidad de viento de $13 \mathrm{~m} / \mathrm{s}$, que es la velocidad de corte de la turbina, al no tener control de pitch. Estas ponen en marcha un sistema de frenado mediante el plegado de las palas o la rotación de la góndola. Lo anterior genera una disminución paulatina de la velocidad angular del rotor, por lo que la potencia tarda en hacerse nula, como se observa en la figura 6 .

En la figura 6 se aprecia que la curva de potencia de la turbina de $7 \mathrm{~kW}$ no se estabiliza. Dado que las pequeñas turbinas suelen ser de autoconsumo, no requieren que su salida sea estable, pues no están obligadas a sincronizarse con la red.

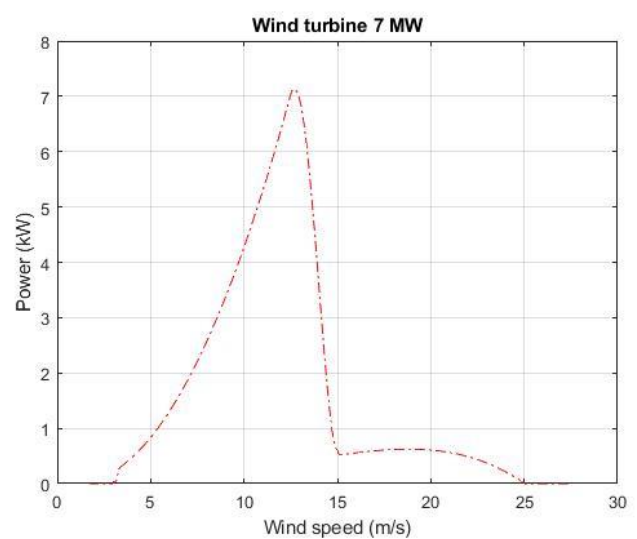

Figura 6: Potencia eléctrica de salida de la turbina de $7 \mathrm{~kW}$.

Al comparar las figuras 5 y 6 se observa que la potencia crece más rápidamente en el aerogenerador de $7 \mathrm{~kW}$, mientras que el de $5 \mathrm{MW}$ presenta un aumento moderado de potencia en velocidades de viento bajas. Esto se debe a que la inercia del aerogenerador de $5 \mathrm{MW}$ es considerablemente mayor al de la pequeña turbina (Tabla 2), lo que genera que oponga más resistencia al arranque.

\section{CONCLUSIONES Y TRABAJOS FUTUROS}

En este trabajo se ha presentado el diseño de una metodología de escalamiento que permite obtener el modelo de un aerogenerador de cierta capacidad a partir de un modelo existente de diferente capacidad. Para ello, se han implementado en MATLAB/Simulink los modelos matemáticos que describen el funcionamiento de un aerogenerador, donde se han identificado los parámetros que definen su potencia nominal. La metodología se diseña como una serie de pasos y un análisis de sensibilidad que deben ser seguidos para lograr el escalamiento de un modelo. La metodología diseñada ha sido implementada para obtener el modelo de una turbina real de $5 \mathrm{MW}$ a partir del modelo de una turbina de 7 $\mathrm{kW}$ con resultados satisfactorios. 
Como trabajos futuros se propone validar estos datos con turbinas grandes offshore, e incorporando la dinámica de estas turbinas

\section{Agradecimientos}

Este trabajo de investigación ha sido desarrollado con financiación del Proyecto MCI AEI/FEDER RTI2018-094902-B-C21, del Ministerio de Ciencia, Innovación y Universidades.

\section{English summary}

\section{MODELING AND SIMULATION OF A 5 MW WIND TURBINE FROM A 7 KW ONE}

\begin{abstract}
In this work, a methodology is proposed to scale the model of a wind turbine of some nominal power to a turbine of different rated power. To do this, the models that describe the operation of a wind turbine are presented and implemented in MATLAB/Simulink. The parameters that define its rated power are identified, and the modifications that must be made to scale one model to another are listed and applied. The simulation results confirm the viability of this methodology, which can be useful to obtain in a systematic and simpler way the model of the most complex large turbines.
\end{abstract}

Keywords: wind energy, wind turbine, modeling and simulation, scaling, electric power.

\section{Referencias}

[1] Burmester, S., Gueydon, S., \& Make, M. (2016). Determination of Scaled Wind Turbine Rotor Characteristics from Three Dimensional RANS Calculations. Journal of Physics: Conference Series, 753(082003), 1

[2] Burton, T., Sharpe, D., Jenkins, N., Bossanyi, E. (2001). Wind energy handbook (Vol. 2). New York: Wiley.

[3] Coutinho Silva, A., Leal dos Santos, C., Diógenes, S., Araújo, A., Corte Real Fernandes, E., Cordeiro de Araújo Bezerra, C., . . . de Andrade, L. (2017). $5 \mathrm{MW}$ and $2 \mathrm{MW}$ NREL wind turbines simulation comparison for steady loading calculations. 24th ABCM International Congress of Mechanical Engineering, 24, 1.

[4] Galvani, P. A., Sun, F., Turkoglu, K. (2016). "Aerodynamic modeling of NREL 5-MW wind Turbine for nonlinear control system design: A case study based on real-time nonlinear receding horizon control”. Aerospace, 3(3), 27.

[5] Heier, S. (2014). Grid integration of wind energy: onshore and offshore conversion systems. John Wiley \& Sons.

[6] Johnson, D. A., Gu, M., Gaunt, B. (2016). "Wind turbine performance in controlled conditions: BEM modeling and comparison with experimental results". International Journal of Rotating Machinery, Article ID 5460823.

[7] Jonkman, J., Butterfield, S., Musial, W., Scott, G. (2009). Definition of a 5-MW reference wind turbine for offshore system development (No. NREL/TP-500-38060). National Renewable Energy Lab.(NREL), Golden, CO (United States).

[8] Khan, J., \& Iqbal, T. (2009). Analysis of a small wind-hydrogen stand-alone hybrid energy system. Applied Energy.

[9] Mikati, M., Santos, M., Armenta, C. (2012) "Modelado y simulación de un sistema conjunto de energía solar y eólica para analizar su dependencia de la red eléctrica." Revista Iberoamericana de Automática e Informática industrial 9(3), 267-281.

[10] Mikati, M., Santos, M., Armenta, C. (2013). "Electric grid dependence on the configuration of a small-scale wind and solar power hybrid system". Renewable energy, 57, 587-593.

[11] NREL, National Wind Technology Center, 1 March 2012. [Online]. Available: https://wind.nrel.gov/forum/wind/viewtopic.php $? \mathrm{t}=582$. [Accessed 2020 December 2020].

[12] Rolan, A., Luna, A., Vazquez, G., Aguilar, D., Azevedo, G. (2009, July). Modeling of a variable speed wind turbine with a permanent magnet synchronous generator. In: 2009 IEEE International Symposium on Industrial Electronics (pp. 734-739). IEEE.

[13] Shabestari, P. M., Mehrizi-Sani, A. (2020). Frequency response improvement of PMSG wind turbines using a washout filter. Energies, 13(18), 4797.

[14] Sierra-García, J. E., Santos, M. (2020). "Performance analysis of a wind turbine pitch neurocontroller with unsupervised learning", Complexity, Article ID 4681767 
[15] Sierra-García, J. E., Santos, M. (2020). "Exploring reward strategies for wind turbine pitch control by reinforcement learning". Applied Sciences, 10(21), 7462.

[16] Tomás-Rodríguez, M., Santos, M. (2019). "Modelling and control of floating offshore wind turbines". Revista Iberoamericana de Automática e Informática Industrial, 16(4), 381390. (C) (1) () 2021 by the authors. access publication under the terms and conditions of the Creative Commons Attribution CC BY-NC-SA 4.0 license (https://creativecommons.org/licenses/byncsa/4.0/deed.e 